\title{
Can observation prevent decay?
}

\section{Failure so far to detect proton decay is not explained by the suggestion that intra-nuclear collisions prevent it. But there is a quantum version of Zeno's paradox to be resolved.}

Although quantum mechanics is more than half a century old, arguments persist about the most elementary features of the theory, even the question of what precisely are the consequences of a measurement of some physical attribute of a quantum system. But did not Heisenberg settle the matter once and for all with his statement of the uncertainty principle, the notion that the uncertainty in the measurement of a pair of canonically conjugate dynamical variables (such as position and momentum, or energy and time) must be reciprocally related in the sense that the product of the two uncertainties is bounded below by Planck's constant? Unfortunately not, as can be told from a row about the lifetime of the proton that has blown up in the pages of Physical Review Letters.

To be sure, it follows from the uncertainty principle that a precise measurement of the momentum of, say, a particle leaves its position essentially undetermined. One of the reasons for the resurgence of interest in the past few years in the theory of quantum measurement is the ambition to extract the utmost information from equipment designed for the measurement of signals virtually buried in receiver noise, the detection of gravitational waves for example. One result is the growth in the past few years of the minor industry concerned with what are known as "quantum non-destruction" measurements. Now, however, an even more elementary issue has bubbled up that of whether it is possible to prevent some phenomenon (say the radioactive decay of an unstable nucleus) from taking place at all by keeping the system (that is, the nucleus) under continuous cbservation.

The argument goes like this. For many quantum systems, for example atoms with less energy than that needed to detach the least strongly bound electron into the energy continuum, most measurements of dynamical quantities yield only one of a set of discrete values, the eigenvalues of the appropriate Schrodinger equation or its equivalent in the Heisenberg matrix formalism. Everybody knows that. So what happens if one measurement is quickly repeated? The effect of the first measurement is not merely to yield as a numerical result one of the possible eigenvalues but also to ensure that the system as a whole is driven into the corresponding eigenstate. So if the measurement is quickly repeated, the result will be exactly the same numerical value.

In the mid-twenties, while the Schrodinger and Heisenberg schools were developing techniques for calculating the allowable eigenvalues for particular quantum systems, Bohr's Copenhagen school was working out the formal language in which these questions are still discussed. At the outset, before any measurements have been made, the state of the system is literally undetermined, some indeterminate mixture of all possible states. The effect of the measurement is to resolve this uncertainty. The Copenhagen word for this process is that of the "preparation" of a system in a particular eigenstate. Once that has been done, the system will stay as it is unless influenced by extraneous forces (such as those occasioned by measurements of a conjugate variable). So if arrangements are made to make some measurement of the state of an unstable nucleus, and to repeat that observation frequently, should not the result be that the system persists in its prepared state and does not decay?

Earlier this year, L.P. Horwitz and E. Katznelson (Phys. Rev. Lett. 50, 1184; 1983) advanced an argument very much like this to suggest that protons bound within nuclei should not decay at anything like the rate suggested by the now fashionable gauge theories of the strong nuclear interaction - and thus to explain the failure so far to detect such decay. What the two authors (from Tel Aviv University, Israel) suggested was that the intranuclear collisions with other nucleons to which any potentially unstable proton must be subjected would ensure its survival in its prepared state - that of a proton. For even though the collisions take place at random intervals, and although the outcome of these collisions is not self-consciously recorded, if a proton should at some instant have decayed (say into a $\pi^{0}$ meson and a positron, one of the more probable decay routes), the result would be that the dynamical behaviour of colliding nucleons would be transformed in such a way as to produce an excited state of the nucleus as a whole. Horwitz and Katznelson therefore argued six months ago that repeated internal collisions would be entirely equivalent to a sequence of measurements repeated at intervals of time comparable with the average time between nucleon collisions. For their temerity, they have brought down on their heads an impressive declaration of dissent (Phys. Rev. Lett. 51, 1599; 1983).

The argument is nevertheless not devoid of interest. The three refutations now published make a number of separate points, of which the most persuasive (nevertheless denied by Horwitz and Katznelson) is that a nucleus is a single entity described by a set of eigenstates whose properties are determined not merely by the constitution of the nucleus but also by the interactions between them. On this view, intranuclear collisions between nucleons are accounted for from the outset, with the consequence that their occurrence is irrelevant to the enumeration of the quantum states in which the nucleus exists, or between which transformations can recur. In passing, however, the counter-arguments have the interest of drawing attention to an often-forgotten feature of decay processes - that while the rate of the decay of a system is usually correctly taken to be a constant, at the outset of the existence of an unstable state there is a short period during which the likelihood of decay is proportional to the square of the elapsed time, which may be put at $A t^{2}$, where $A$ is a constant and $t$ is elapsed time. Then the probability that the system will not have decayed is $\left(1-A t^{2}\right)$ and, if it is imagined that a large number of measurements, say $N$, is carried out at intervals of $t$, the probability that the system will not have decayed after an elapsed time of $\mathrm{N} t$ is $\left(1-A t^{2}\right)^{N}$, which works out as $\exp \left(-A T^{2} / N\right)$ where $T$ is the total elapsed time $\mathrm{N} t$ and $N$ is assumed large. Since $N$ may be arbitrarily large, the chance of a system not having decayed after the passage of time $T$ may be made arbitrarily close to unity.

Kevin Cahill of the University of New Mexico, one of the tormentors of Horwitz and Katznelson, points to this paradoxical conclusion before going on to argue that the paradox is unreal because the collisions do not constitute observations. But can the paradox be real? Coincidentally, but apparently without the knowledge of Horwitz and Katznelson and their critics, the question has been taken up by $\mathrm{M}$. Bunge and $\mathrm{A}$. J. Kalnay in Il Nuevo Cimento (B77, 10; 1983), who explicitly deal with the general suspicion that the paradox must be a fraud. Their conclusion is simple - the false assumption in the calculation summarized above is that measurements can be carried out instantaneously. In reality, this cannot be so - and the process of measurement can be as much a stimulus to transformation as to stasis.

John Maddox 